\title{
Altair's inclination from line profile analysis ${ }^{\star}$
}

\author{
A. Reiners ${ }^{1,2}$ and F. Royer ${ }^{3,4}$ \\ 1 Astronomy Department, University of California, Berkeley, CA 94720, USA \\ e-mail: areiners@astron.berkeley.edu \\ 2 Hamburger Sternwarte, Universität Hamburg, Gojenbergsweg 112, 21029 Hamburg, Germany \\ 3 Observatoire de Genève, 51 chemin des Maillettes, 1290 Sauverny, Switzerland \\ e-mail: frederic.royer@obs.unige.ch \\ ${ }^{4}$ GEPI, CNRS UMR 8111, Observatoire de Paris, 5 place Janssen, 92195 Meudon Cedex, France
}

Received 18 May 2004 / Accepted 9 August 2004

\begin{abstract}
We present a detailed spectroscopic study of line broadening in the A7IV-V star Altair. In a wavelength region covering $690 \AA$ we reconstruct the overall broadening profile taking into account more than 650 spectral lines. From the broadening profile we determine the projected rotational velocity $v \sin i$, derive an upper limit for the equatorial velocity $v$ from the shape of the profile and search for signatures of differential rotation. Our redetermined value of $v \sin i$ is $(227 \pm 11) \mathrm{km} \mathrm{s}^{-1}$. Measuring the first two zeros of the Fourier transformed broadening profile yields no signatures of differential rotation. We derive that Altair is seen under an inclination angle higher than $i=68^{\circ}$ and it rotates at $v<245 \mathrm{~km} \mathrm{~s}^{-1}$ or slower than 53\% of breakup velocity on a $1 \sigma$ level.
\end{abstract}

Key words. stars: rotation - stars: early-type - line: profiles - stars: individual: Altair

\section{Introduction}

As the 12th brightest star in the sky, Altair ( $\alpha$ Aql, HR 7557, HD 187642) has been extensively studied. Recently Buzasi et al. (2004) listed most of its physical parameters in their introduction. Altair is an A7IV-V main sequence star known to be a fast rotator with a projected rotational velocity $v \sin i=$ $217 \mathrm{~km} \mathrm{~s}^{-1}$ (Royer et al. 2002). With a distance of $5.14 \mathrm{pc}$ (ESA 1997) it is one of the nearest bright stars, allowing van Belle et al. (2001) to measure the oblateness of the star using interferometric observations. They derived an axial ratio $a / b=1.140 \pm 0.029$ and a value for the $v \sin i$ of $210 \pm 13 \mathrm{~km} \mathrm{~s}^{-1}$. Interferometry also allows putting constraints on stellar inclination - a parameter usually not available from standard spectroscopic techniques. Their determination of the inclination $i$ of the rotational axis gives a value in the range of $30^{\circ}$ to $90^{\circ}$ within $1 \sigma$ (van Belle et al. 2001, Fig. 5).

Spectroscopic techniques are usually not sensitive to inclination in the "classical" case of spherical stars. On the contrary, for fast rotators, the stellar surface is distorted by centrifugal forces and line profiles depend on the angle between the rotational axis and the line of sight. Moreover, in such objects the effective temperature varies with gravity over the stellar surface: this is the gravity darkening (von Zeipel 1924).

* Based on observations carried out at Observatoire de HauteProvence (OHP), at the German-Spanish Astronomical Centre, Calar Alto, and retrieved from the Elodie Archive at Observatoire de HauteProvence (OHP).
Scrutinizing the shape of individual lines, Gulliver et al. (1994) determined the inclination angle of Vega from spectroscopic data. In a more general context, Reiners (2003) showed the effects of inclination and gravity darkening on line profiles of fast rotators in the Fourier domain. He showed that in spectra of fast rotators the unprojected rotational velocity $v$ is directly measurable from the line shape. It is the aim of this paper to put stronger constraints on the inclination of Altair from spectroscopic measurements by measuring its (unprojected) equatorial velocity and $v \sin i$.

\section{Data}

\subsection{Altair data}

Altair was observed on October 1st 2003 with the ÉLODIE échelle spectrograph (Baranne et al. 1996) at the Observatoire de Haute-Provence. The wavelength coverage spans 3850 to $6800 \AA$, with a resolving power of 42000 . Five single exposures were taken; details of the observational data are listed in Table 1.

The orders of the échelle spectrum were merged using the method by Erspamer \& North (2002). The barycentric radial velocity correction varies by about $0.025 \mathrm{~km} \mathrm{~s}^{-1}$ in the short time interval of the observations, and the peculiar radial velocity of Altair is not expected to change significantly. The comparison of wavelength positions for telluric lines $\left(\mathrm{O}_{2}\right)$ ensures that no significant instrumental wavelength shift occurred 
Table 1. The collected spectra for each stellar target, giving the corresponding spectrograph, the resolving power, the Julian date (HJD) and signal-to-noise ratio $(S / N)$ for each observation. The $S / N$ for ÉLODIE spectra is estimated at $5550 \AA$. Values of $v$ sin $i$ are from Royer et al. (2002) and given in $\mathrm{km} \mathrm{s}^{-1}$.

\begin{tabular}{llcrccc}
\hline \hline Object & Sp. type & Instrument & $\lambda / \Delta \lambda$ & HJD & $S / N$ & $v \sin i$ \\
\hline Altair & A7IV/V & ÉLODIE & 42000 & 2452914.2574 & 180 & $\sim 220$ \\
& & & & 2452914.2600 & 200 & \\
& & & & 2452914.2628 & 280 & \\
& & & & 2452914.2655 & 230 & \\
& & & & 2452914.2683 & 250 & \\
\hline HD 27819 & A7IV/V & ÉLODIE & 42000 & 2450499.4221 & 180 & $\sim 40$ \\
\hline$\gamma$ Boo & A7III/IV & FOCES & 40000 & 2452336.0736 & 300 & $\sim 130$ \\
\hline HD 118623 & A7III & FOCES & 40000 & 2452332.1416 & 300 & $\sim 210$ \\
\hline
\end{tabular}

between the consecutive exposures, and the merged spectra are therefore simply co-added.

\subsection{Calibration data}

We used spectra of three stars of similar spectral type to calibrate our measurements of Altair's spectrum. Spectra observed with the FOCES spectrograph at the $2.2 \mathrm{~m}$ telescope at the German-Spanish Astronomical Centre, Calar Alto, were taken in February 2002; for details on the reduction cf. Reiners \& Schmitt (2003). The spectrum of HD 27819 was taken from the ÉLODIE Archive ${ }^{1}$. The calibration stars are listed in Table 1.

\section{Continuum normalization}

For the analysis of spectral line shapes the determination of the continuum flux is crucial. In the spectra of stars with rotation rates as high as Altair's, spectral lines are severely blended and the flux hardly reaches the continuum in any spectral region. Thus, normalizing Altair's spectrum by eye-guided continuum fitting would always assume too low a continuum which significantly affects the shape of the line profiles.

We thus took high quality spectra of other stars of similar spectral types, some of them having considerably lower rotation rates; in their spectra the continuum is nicely visible. From the FOCES campaign we took two stars; HD 118623 is an A7III with a rotational velocity very similar to Altair's. $\gamma$ Boo has a rotational velocity of $v \sin i \sim 130 \mathrm{~km} \mathrm{~s}^{-1}$, i.e. significantly lower than that of Altair and HD 118623. In the spectrum of $\gamma$ Boo the flux reaches the continuum level in some regions and continuum fitting is possible.

The spectrum of $\gamma$ Boo was normalized by fitting the regions where the flux reaches the continuum level. We also used this spline fit to normalize the spectrum of HD 118623 which was taken during the same observing campaign in the same instrument setup. The coincidence of their non-normalized spectra is shown in Fig. 1, supporting the use of identical normalization functions.

With the normalized spectrum of HD 118623, a star similar to Altair with respect to spectral type and projected rotational

\footnotetext{
${ }^{1}$ http://atlas.obs-hp. fr/elodie/
}

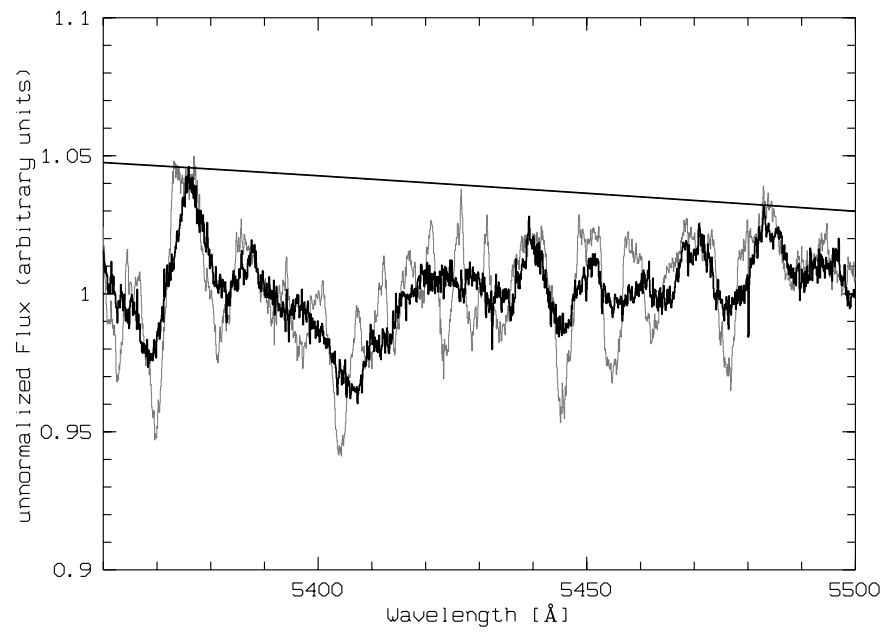

Fig. 1. A small region of the non-normalized spectra of $\gamma$ Boo (grey) and HD 118623 (black). The fit to the assumed continuum is overplotted and is in good agreement with both spectra.

velocity, we could then normalize the spectrum of Altair by matching its overall spectral shape to that of HD 118623. Both normalized spectra are shown in Fig. 2 in the whole wavelength region we used in the following.

\section{Reconstructing the global broadening function}

Spectra of stars rotating as fast as Altair are completely dominated by rotational broadening. Temperature broadening and turbulent motions are about two orders of magnitude smaller than rotational broadening. Rotation affects all spectral lines in the same characteristic manner. However, the assumption we have to make is that the line profiles of individual lines do not vary significantly (at least not systematically) across the stellar disk. For individual lines this is not true, but if hundreds of lines of different species are used, variations among different lines are expected to level out. What one gets is a profile that reflects the mean rotational broadening affecting all lines. This method is in contrast with using individual lines (e.g., Gulliver et al. 1994) and line-specific variations with temperature or gravity are not taken into account. For Altair, systematic effects are not expected to be as severe as, e.g., for Vega. We will discuss this in Sect. 5.1. However, individual line profiles are severely 


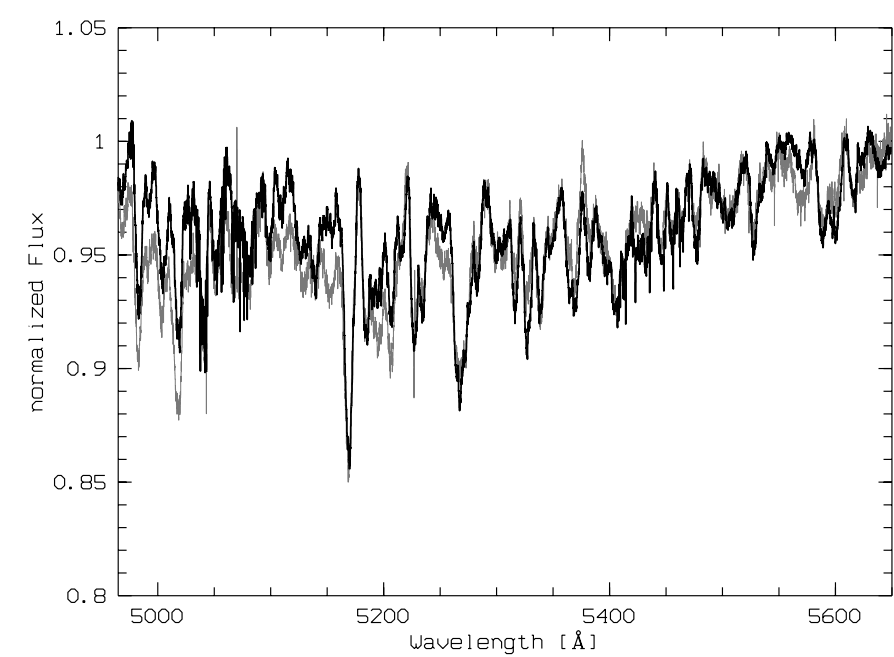

Fig. 2. Normalized spectra of HD 118623 (grey) and Altair (black). The whole region used to derive Altair's broadening function is shown.

blended in Altair's spectrum, making studies of individual lines difficult and we think that recovering line broadening from as many lines as possible yields the most reliable information on stellar rotation.

The reconstruction of Altair's mean broadening profile is done by a deconvolution algorithm. The algorithm searches the function that gives the best fit to the data when it is convolved with a given template. The template is required to be a precise spectrum of the star without rotation (neglecting other broadeners). Since no such template is available we usually try to simultaneously fit the line strengths, i.e., equivalent widths, of all spectral lines (cf. Reiners \& Schmitt 2003). In the case of Altair, however, the blending of the large amount of spectral lines due to the high rotational velocity is so severe, that simultaneously fitting the broadening function and line strengths is not possible. We thus did not determine the template from the spectrum of Altair but from the spectrum of a star of similar spectral type.

In spectra of slower rotators with less severe blending, the broadening function and line strengths can successfully be fitted simultaneously. From the ÉLODIE Archive we took a spectrum of HD 27819, a A7IV-V star rotating at $v \sin i \sim$ $40 \mathrm{~km} \mathrm{~s}^{-1}$. Normalization was made by eye using the clearly visible continuum. A comparison of the normalized spectra of Altair and HD 27819 is shown in Fig. 3; the spectral similarity is clearly visible. For this "slow" rotator HD 27819 the broadening function and line strengths were derived iteratively from the spectrum using our deconvolution algorithm (cf. Reiners $\&$ Schmitt 2003). We used the whole wavelength region between $\lambda=4960 \AA$ and $\lambda=5650 \AA$. In this spectral region all lines are dominated by rotation, i.e., hydrogen lines are not contained in the region. Roughly 650 spectral lines were used in the fit. Wavelength information was taken from the Vienna Atomic Line Database (Kupka et al. 1999, VALD).

The result of this procedure is shown in Fig. 4 where we plot the result of convolving the recovered broadening function - shown in the inset - with the optimized template. Note that

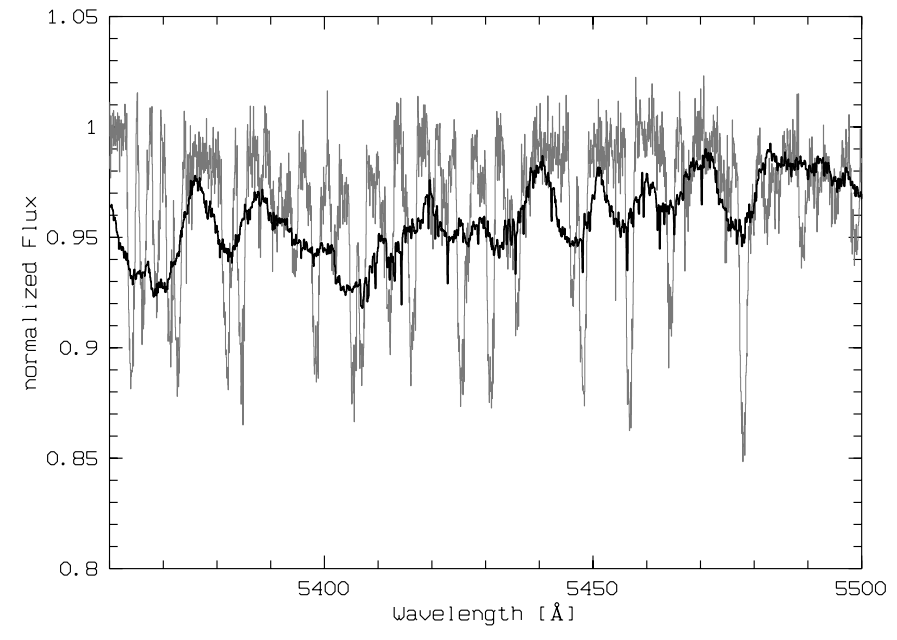

Fig. 3. Comparison of the normalized spectra of HD 27819 (grey line) and Altair (black line) in a small spectral window.

each pixel of the broadening function is a free parameter in the algorithm and that the broadening function resembles nicely symmetric rotational broadening (from the profile we derive a $v \sin i$ of $46 \mathrm{~km} \mathrm{~s}^{-1}$ ). The data (grey line) are reproduced in great detail; since individual lines are not severely blended, ambiguities between the shape of the broadening function and line strengths are very small.

For the deconvolution of Altair's broadening profile we now used the same optimized template, i.e., the same line strengths as derived for HD 27819. Note that the observed spectrum of HD 27819 could not be used as a template since it already is broadened by $46 \mathrm{~km} \mathrm{~s}^{-1}$ and a convolution of two rotational broadening functions does not yield a third one. From the normalized spectrum of Altair we finally derived the profile that gives the smallest chi-square when convolved with this delta-like template.

A small region of the result is shown in Fig. 5; the quality of the fit is representative for the whole wavelength region. The derived broadening function is shown in the inset. Note that no additional changes were made to the continuum and that the broadening function is nicely symmetric. A small linear slope in the overall appearance might be visible. While this slope is likely due to a slight continuum mismatch, it does not significantly affect the line shape. The pixel size used is only a fraction of the instrument's resolution and the small jitter in the broadening function is numerical. The amount of jitter corresponds to a signal-to-noise ratio $(S / N)$ of well over 1000 . We calculated the variance of each pixel in the chi-square fit with regard to the two nearest neighbors of each pixel. This yields an $S / N$ of several thousands. Unfortunately, the uncertainties in the broadening function are dominated by our lack of knowledge of the continuum and by systematically incorrect line strengths (in detail, line strengths of Altair are definitely different from those of HD 27819). In what follows we estimate a rather conservative $S / N$ of 1000 as the " $1 \sigma$ "-level. Consequently we use an $S / N$ of 500 for our calculations of $2 \sigma$ borders. In the left panel of Fig. 6 the global broadening function of Altair is plotted with error bars corresponding to an $S / N$ of 1000 . 


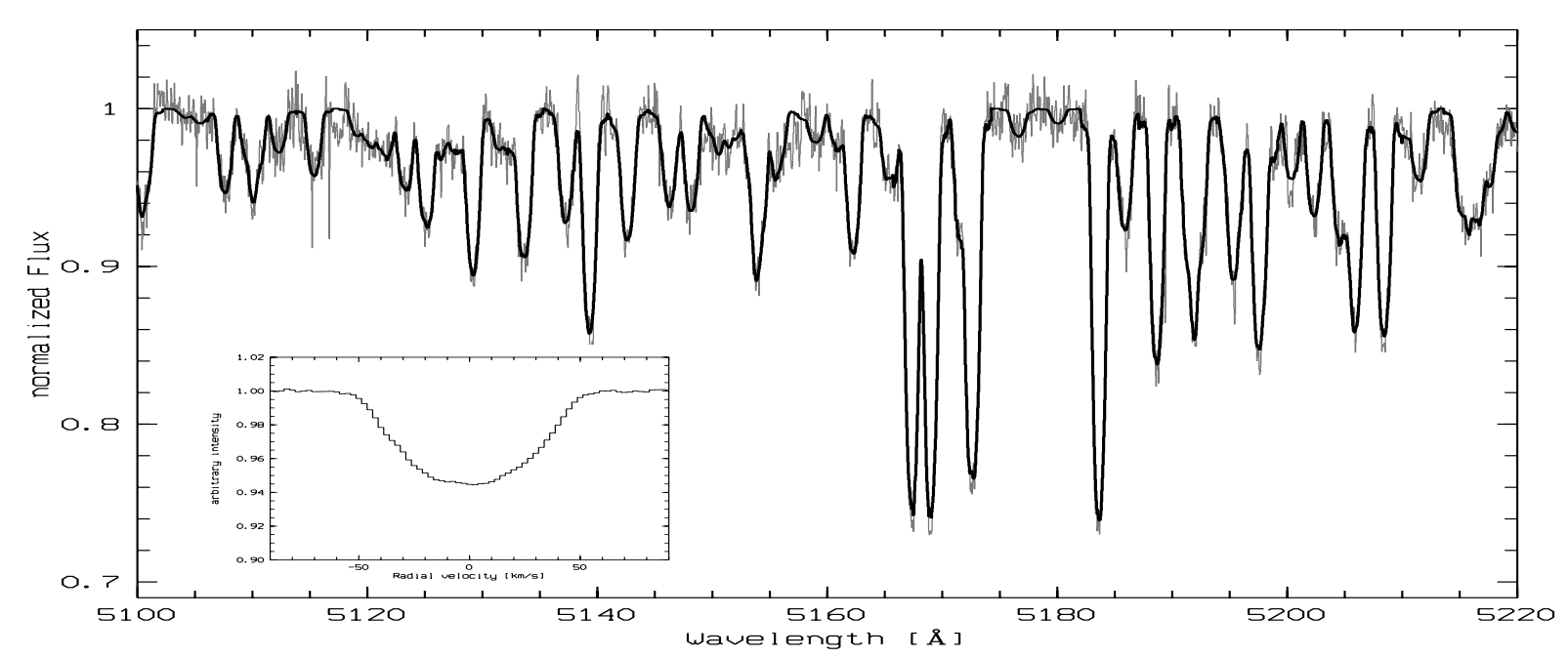

Fig. 4. Data (grey) and fit (black) achieved from our deconvolution algorithm for HD 27819. The quality of the fit is typical for the whole wavelength region. The deconvolved "global" profile is plotted in the inset.

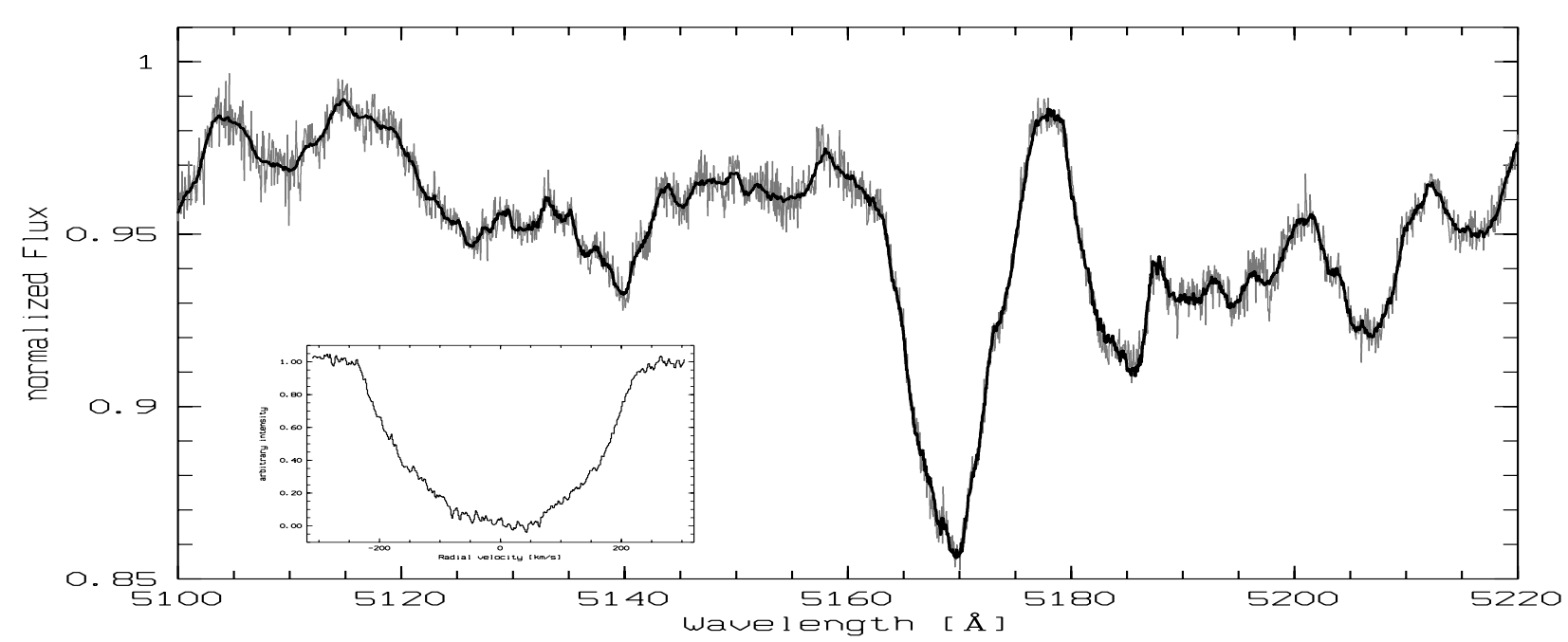

Fig. 5. Data (grey line) and achieved fit (black line) of Altair. Fit quality is typical for the whole wavelength region. The deconvolved "overall" profile is plotted in the inset.

The global broadening function shows small asymmetries. From our analysis we cannot say whether these asymmetries are real or due to incorrect line strengths in the template. However, within the error bars the broadening function is symmetric, i.e. the broadening function mirrored at the center falls withing the error bars.

In the right panel of Fig. 6 the Fourier transform of the derived broadening function is shown with the respective $1 \sigma$ error bars. The signal can clearly be followed to the third sidelobe before it reaches the noise level. In the calculation of $v \sin i$ and $i$ only the positions of the first two zeros are needed; apparently both are well defined.

\section{Spectroscopic rotation and inclination}

Studying the characteristics of rotational broadening, zeros of the Fourier transform of the broadening profile are convenient observables (cf. Gray 1992). Since convolutions become multiplications in the Fourier domain, especially zeros inferred from any broadening mechanism remain unchanged by other effects like turbulence etc.; the intensity is changed - the position of the zeros is not. For a star rotating as fast as Altair, no effects are expected that infer signatures at frequencies as low as those inferred by rotation. Thus, measuring Fourier transform zeros is a very robust method to access rotational broadening and the zeros can easily and precisely be determined. The first zero is frequently used to determine stellar projected rotational velocities $v \sin i$, as shown in Dravins et al. (1990) for the case of linear limb darkening.

The effects of rotation and inclination on absorption line profiles have been studied by Reiners (2003). He shows that the ratio $q_{2} / q_{1}$ of the first two zeros $q_{1}$ and $q_{2}$ is sensitive to very fast rotation. He concludes that the ratio $q_{2} / q_{1}$ does not depend on the value of $v \sin i$, but on the equatorial velocity $v$ regardless of the inclination angle $i$ (and on the rotation law, 

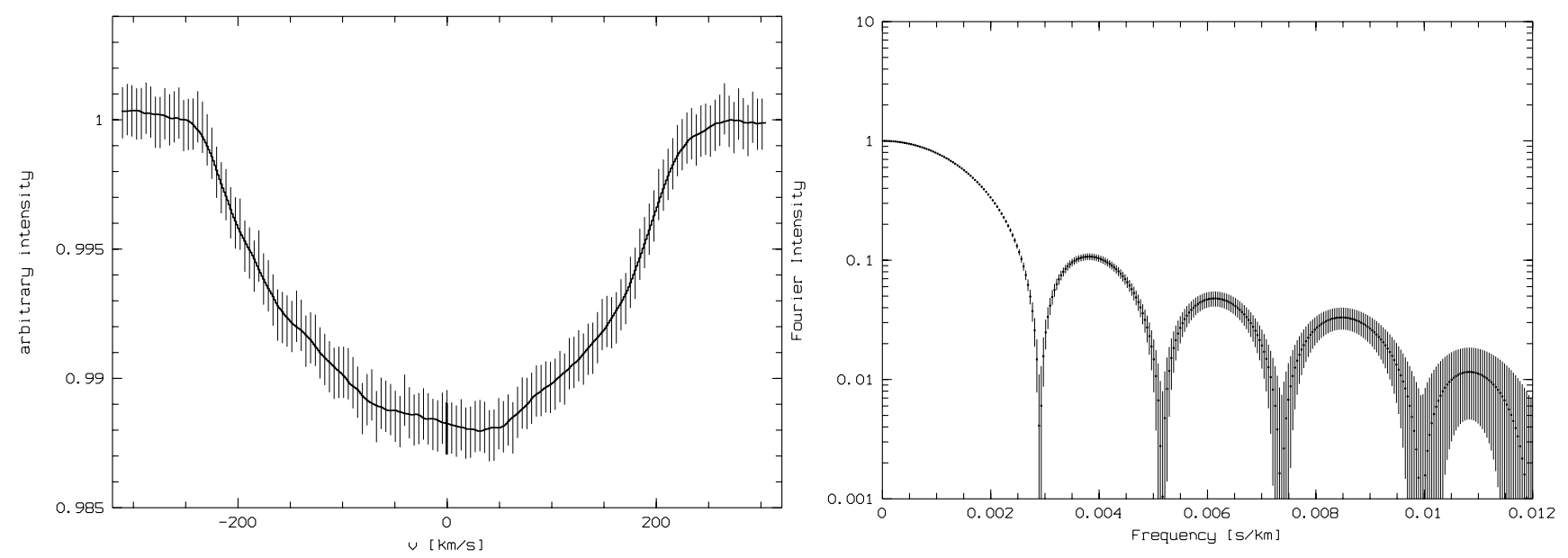

Fig. 6. Left panel: global broadening function of Altair reconstructed from about 650 lines. Error bars corresponding to a signal-to-noise ratio of 1000 are overplotted. Right panel: Fourier transform of the reconstructed broadening function (left panel) with its error bars. Note the logarithmic scale on the $y$-axis.

Table 2. Measured values of Fourier transform zeros and calculated parameters $v \sin i, v$ and $i$. For $v \sin i$ formal and estimated systematic errors are given. For $1 \sigma$ and $2 \sigma$ confidence levels in $v$ and $i$, signal-tonoise ratios of the broadening function of 1000 and 500 are adopted, respectively.

\begin{tabular}{cc}
\hline \hline Parameter & Value \\
\hline$q_{1}$ & $(2.91 \pm 0.03) \times 10^{-3} \mathrm{~s} \mathrm{~km}^{-1}$ \\
$q_{2}$ & $(5.16 \pm 0.08) \times 10^{-3} \mathrm{~s} \mathrm{~km}^{-1}$ \\
$\frac{q_{2}}{q_{1}}$ & $1.77 \pm 0.03$ \\
\hline$v \sin i$ & $(227 \pm 2 \pm 11) \mathrm{km} \mathrm{s}^{-1}$ \\
$v$ & $<245 \mathrm{~km} \mathrm{~s}^{-1} \quad(1 \sigma)$ \\
& $<305 \mathrm{~km} \mathrm{~s}^{-1} \quad(2 \sigma)$ \\
$i$ & $>68^{\circ} \quad(1 \sigma)$ \\
& $>45^{\circ} \quad(2 \sigma)$ \\
\hline
\end{tabular}

see Sect. 5.2). Thus measuring $v \sin i$ from $q_{1}$ and $v$ from the ratio $q_{2} / q_{1}$ yields the value of the inclination angle $i$ !

By modeling absorption profiles of fast rotators, Reiners (2003) finds that the ratio $q_{2} / q_{1}$ can be expressed as

$q_{2} / q_{1}=1.75+a v+b v^{2}$.

Values of $a$ and $b$ are given for different stellar models in Table 1 of that paper. The mechanism determining the values of $a$ and $b$ is gravity darkening, described by the parameter $\beta$ in

$T_{\text {eff }} \propto g^{\beta}$,

with $g$ the stellar surface gravity. For Altair we get a value of $\beta \sim 0.09$ from the tables given in Claret (1998), i.e. the results calculated by Reiners \& Schmitt (2003) for the case of an F0 star are directly applicable to Altair. Thus, for the parameters in Eq. (1) we find $a=.740 \times 10^{-4}$ and $b=-.345 \times 10^{-6}$.

Our measurements of the first two zeros $q_{1}$ and $q_{2}$ of the Fourier transform of the broadening profile are given in Table 2. Assuming a linear limb darkening law with a limb darkening parameter $\epsilon=0.6$ we calculate Altair's projected rotational velocity as $v \sin i=227 \pm 2 \mathrm{~km} \mathrm{~s}^{-1}$. Note that the formal error due to the uncertainty of $q_{1}$ is given. We estimate the systematic error in $v \sin i$ to be about $5 \%$ due to continuum and line strength uncertainties (cf. Reiners \& Schmitt 2003). From Eq. (1) we can calculate Altair's equatorial velocity $v$ from the ratio $q_{2} / q_{1}$.

In the left panel of Fig. $7 v$ is plotted as a function of $q_{2} / q_{1}$ with our measurement of $v \sin i$ overplotted as a horizontal line, and the measurement of $q_{2} / q_{1}$ overplotted with its $1 \sigma$ and $2 \sigma$ error levels as vertical lines. The plot shows that $q_{2} / q_{1}$ is generally expected to be smaller than 1.765 , i.e. the measured value of $q_{2} / q_{1}=1.77$ is not expected for any possible inclination angle. However, within the $1 \sigma$ region we find possible values of $v\left(q_{2} / q_{1}\right)$ which are also consistent with the measured value of $v \sin i$.

Calculating Altair's inclination angle $i$ from the measured $v \sin i$ and $v\left(q_{2} / q_{1}\right)$ we find $i\left(q_{2} / q_{1}\right)$. We plot $i\left(q_{2} / q_{1}\right)$ in the right panel of Fig. 7. $1 \sigma$ and $2 \sigma$ confidence levels in $q_{2} / q_{1}$ and systematic errors of $5 \%$ in $v \sin i$ are indicated by dashed lines. We find that our measurements of $v \sin i$ and $q_{2} / q_{1}$ are consistent within $1 \sigma$ for values of $i>68^{\circ}$.

\subsection{Intrinsic line variations}

Variations of line profiles and line strengths have not been taken into account in our procedure of line profile reconstruction. For the case of Vega (A0V), Gulliver et al. (1994) showed that for the two lines they used line-intrinsic effects dominate the line profile; they observe a flattened profile instead of a cuspy shape. The difference with our method is twofold: (i) gravity darkening as expressed in terms of the parameter $\beta$ (Eq. (2)) is only 0.09 for Altair while it is 0.25 for Vega. Temperature variations with gravity are thus much weaker in the case of Altair; (ii) our reconstruction procedure uses about 650 lines to determine the shape of the line profile; we get a weighted mean of 650 line profiles. Although we do not know specific temperature dependencies for all lines, we do not expect them to be systematic. Studying temperature and gravity dependence of line strengths and shapes for different spectral types would significantly reduce the systematic errors but is beyond the scope 

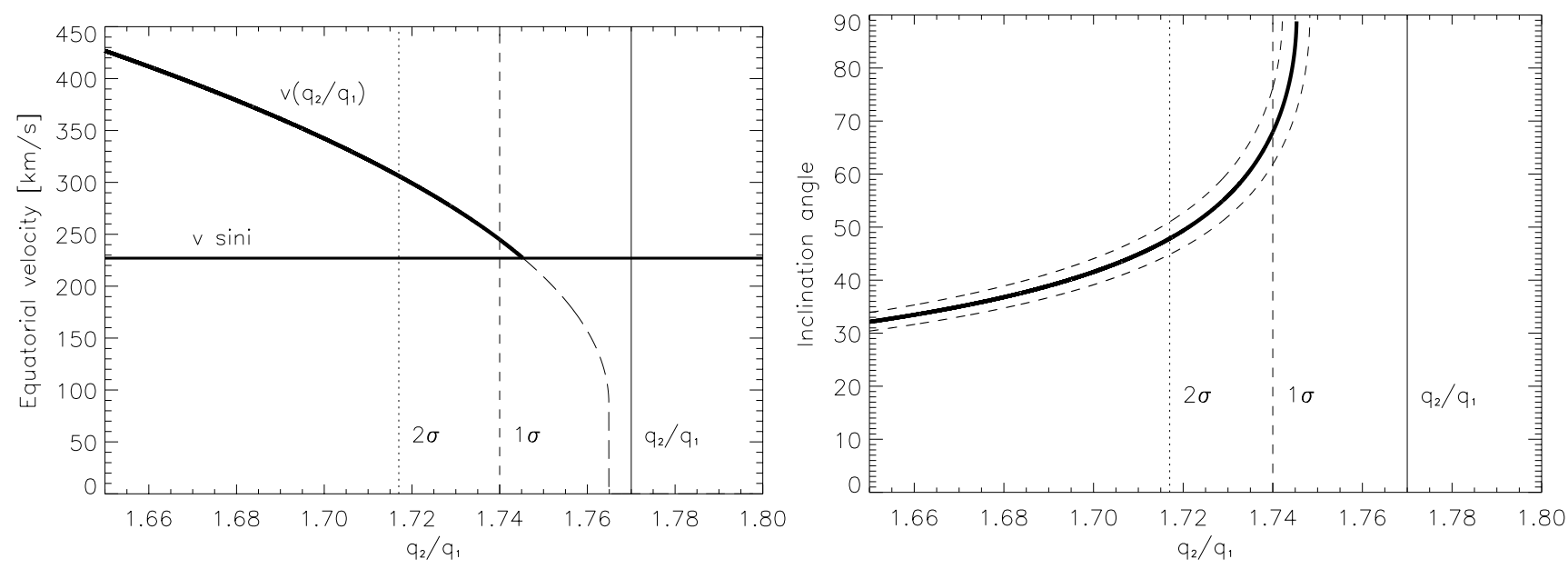

Fig. 7. Left panel: equatorial velocity $v$ as a function of $q_{2} / q_{1}$ according to Eq. (1). Vertical lines indicate measured values of $q_{2} / q_{1}$ and its confidence levels, the horizontal line shows the measured $v \sin i$. The allowed region where $v>v \sin i$ is plotted as a thick line. Right panel: inclination angle $i$ (in degree) of Altair as function of $q_{2} / q_{1}$ (solid thick line). Measurements of $q_{2} / q_{1}$ are plotted as in the left panel. Dashed lines indicate the region allowed taking into account the systematic errors of $v \sin i$.

of this paper. For this study we estimate the systematic errors as given below and leave the detailed study for a forthcoming publication.

\subsection{Differential rotation?}

It has been argued that Altair might undergo substantial surface differential rotation since it was found to be an X-ray source (Ferrero et al. 1995). The parameter $q_{2} / q_{1}$ is also a sensitive indicator for solar-like surface differential rotation with the equator rotating at a higher velocity than the polar regions do. In the case of differential rotation, $q_{2} / q_{1}$ is expected to be significantly smaller that 1.75 which is not the case for Altair. We thus conclude that no signatures of solar-like differential rotation could be found in the line profiles of Altair, i.e. solarlike differential rotation is not stronger than $10 \%$ (Reiners \& Schmitt 2002). However, Altair is at least two orders of magnitude fainter in X-rays than active F-stars are. Even if a magnetic dynamo were the source for this faint X-ray flux, differential rotation may be below the detection limit.

\section{Conclusions}

We have spectroscopically determined constraints on Altair's inclination angle and differential rotation from the global rotational broadening profile derived from about 650 spectral absorption lines. The measured observables and our results are summarized in Table 2 where values of equatorial velocity $v$ and inclination angle $i$ are given with their respective $1 \sigma$ and $2 \sigma$ levels.

No signatures of surface differential rotation were detected, i.e. solar-like differential rotation stronger than $10 \%$ can be ruled out.

From the measured zeros of the Fourier transformed broadening profile we conclude that Altair's equatorial velocity is not faster than $245 \mathrm{~km} \mathrm{~s}^{-1}\left(305 \mathrm{~km} \mathrm{~s}^{-1}\right)$ or $53 \%$ (72\%) of breakup velocity $v_{\text {crit }}=426 \pm 12 \mathrm{~km} \mathrm{~s}^{-1}$ (van Belle et al. 2001). Thus its rotation axis is seen under an inclination angle larger than $68^{\circ}$ on a $1 \sigma$-level ( $45^{\circ}$ on a $2 \sigma$-level). These results put additional constraints on the possible rotation rate and inclination angle of Altair as determined from interferometric data (van Belle et al. 2001).

Acknowledgements. We profoundly thank Dr A. Lèbre who collected Altair's spectra at Observatoire de Haute-Provence. This work was partly supported by the Swiss National Science Foundation. A.R. acknowledges financial support from the Deutsche Forschungsgemeinschaft under grant DFG-SCHM 1032/10-2 and has received research funding from the European Commission's Sixth Framework Programme as an Outgoing International Fellow (MOIFCT-2004-002544).

\section{References}

Baranne, A., Queloz, D., Mayor, M., et al. 1996, A\&AS, 119, 373 Buzasi, D. L., Bruntt, H., Bedding, T. R., et al. 2004, ArXiv Astrophysics e-prints

Claret, A. 1998, A\&AS, 131, 395

Dravins, D., Lindegren, L., \& Torkelsson, U. 1990, A\&A, 237, 137

Erspamer, D., \& North, P. 2002, A\&A, 383, 227

ESA 1997, The Hipparcos and Tycho Catalogues, ESA-SP, 1200

Ferrero, R. F., Gouttebroze, P., Catalano, S., et al. 1995, ApJ, 439, 1011

Gray D.F. 1992, The observation and analysis of stellar photospheres (Cambridge: Cambridge Univ. Press)

Gulliver, A. F., Hill, G., \& Adelman, S. J. 1994, ApJ, 429, L81

Kupka, F., Piskunov, N. E., Ryabchikova, T. A., Stempels, H. C., \& Weiss, W. W. 1999, A\&AS, 138, 119

Reiners, A. 2003, A\&A, 408, 707

Reiners, A., \& Schmitt, J. H. M. M. 2002, A\&A, 384, 155

Reiners, A., \& Schmitt, J. H. M. M. 2003, A\&A, 412, 813

Royer, F., Grenier, S., Baylac, M.-O., Gómez, A. E., \& Zorec, J. 2002, A\&A, 393, 897

van Belle, G. T., Ciardi, D. R., Thompson, R. R., Akeson, R. L., \& Lada, E. A. 2001, ApJ, 559, 1155

von Zeipel, H. 1924, MNRAS, 84, 665 